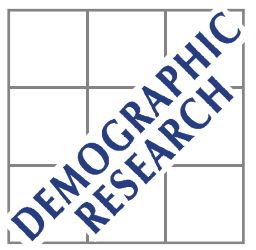

Demographic Research a free, expedited, online journal of peer-reviewed research and commentary in the population sciences published by the Max Planck Institute for Demographic Research Konrad-Zuse Str. 1, D-18057 Rostock · GERMANY www.demographic-research.org

DEMOGRAPHIC RESEARCH

VOLUME 26, ARTICLE 9, PAGES 191-206

PUBLISHED 13 MARCH 2012

http://www.demographic-research.org/Volumes/Vol26/9/

DOI: $10.4054 /$ DemRes.2012.26.9

Research Article

\title{
Discussing the Strehler-Mildvan model of mortality
}

\section{Maxim Finkelstein}

\section{(C) 2012 Maxim Finkelstein.}

This open-access work is published under the terms of the Creative Commons Attribution NonCommercial License 2.0 Germany, which permits use,

reproduction \& distribution in any medium for non-commercial purposes, provided the original author(s) and source are given credit.

See http:// creativecommons.org/licenses/by-nc/2.0/de/ 


\section{Table of Contents}

1 Introduction 192

2 Assumptions and basic relationships 193

3 What 'drives' the SM correlation? 196

4 The Strehler-Mildvan model and mortality deceleration 197

$5 \quad$ An alternative approach: Lifesaving $\Rightarrow$ proportional hazards $\quad 199$

6 Discussion 201

7 Acknowledgements 203

$\begin{array}{ll}\text { References } & 204\end{array}$ 


\title{
Discussing the Strehler-Mildvan model of mortality
}

\author{
Maxim Finkelstein ${ }^{1}$
}

\begin{abstract}
BACKGROUND

Half a century ago, Strehler and Mildvan (1960) published a seminal paper that, based on certain assumptions (postulates), theoretically 'justified' the Gompertz law of mortality.
\end{abstract}

\section{OBJECTIVE}

We intend to discuss the assumptions and limitations of the original Strehler-Mildvan model (as well as of the Strehler-Mildvan correlation), and to consider some modifications and departures from this model.

\section{METHODS}

We use the framework of stochastic point processes for analyzing the original StrehlerMildvan model. We also suggest using the 'lifesaving approach' to describe the departure from rectangularization due to the shifts in the survival curves for human mortality that were observed in the second half of the previous century.

\section{RESULTS}

We show that the Strehler-Mildvan model can be justified only under the additional assumption that the process of shocks (demands for energy) follows the Poisson pattern. We also suggest a modification that accounts for the oldest-old mortality plateau.

${ }^{1}$ Department of Mathematical Statistics, University of the Free State, South Africa. Max Planck Institute for Demographic Research, Rostock, Germany. E-mail: FinkelM@ufs.ac.za. 


\section{Introduction}

The Strehler-Mildvan (1960) model suggests the justification of an exponential increase in the force of mortality $\mu(t)$, and describes some formal properties of the Gompertz mortality curve (Gompertz 1825):

$$
\mu(t)=a e^{b t}
$$

The conventional generalization is the Gompertz-Makeham model (Makeham 1860), which adds a constant term $c$ to the right hand side of (1) in order to account for the 'background' mortality. In the current paper, as in the original publication, we will assume that this term is negligible, and we will discuss this assumption in Section 2. Equation (1) usually provides a satisfactory fit to human mortality data for the ages from maturity to the upper limit of around 90-100 years.

The goal of this note is to discuss the underlying assumptions of the StrehlerMildvan (SM) model and the SM correlation, which defines a negative correlation between parameters $a$ and $b$. For several decades, the SM correlation was believed to be a universal demographic law valid for both period and cohort mortality data (Yashin et al. 2001). However, some departures from this model have also been observed, and some possible causes for these deviations are considered here.

We also suggest a generalization of the SM model, based on replacing the Gompertz curve (1) by the corresponding 'logistic-type mortality curve', which accounts for the mortality plateau for human populations obtained via the gammaGompertz frailty model (Vaupel et al. 1979).

The SM model relies on the notion of vitality; i.e., an organism is characterized by its vitality function $V(t), V(0) \equiv V_{0}$, which decreases with age $t$. An alternative, vitality-independent approach is suggested in Section 5. This approach is based on the concept of 'lifesaving', and applies the stochastic model, suggested in Finkelstein (2005), that is used to explain the possible reasons for departures from the rectangularization pattern of the survival curve observed for human populations in the past half-century. Finally, we wish to mention that our intention in this short note is not to perform a comprehensive review of the relevant literature, but rather to refer to the sources that are necessary and important for our discussion. 


\section{Assumptions and basic relationships}

According to Strehler and Mildvan (1960), an organism is subject to stresses of an internal or an external nature that lead to demands for energy. Let $\left(T_{i}, Y_{i}\right), i=1,2, \ldots$ be the sequence of pairs of i.i.d. random variables (therefore, the notation will be $(T, Y)$ ), characterizing the times at which stress events (demands for energy) occur, and the value of the demand for energy from an organism that is needed to recover from these stresses, respectively. Let $K(t)$ be the rate of the corresponding counting process describing the arrival times of stress events. The following assumptions are made:

\section{Assumption 1}

$Y_{i}$ are exponentially distributed:

$$
P(Y>y)=e^{-\frac{y}{D}},
$$

where, $D$ is the mean value of this demand.

Why exponentially? No real justification is given in the original paper, except for the explanation that the Maxwell-Boltzmann distribution of molecules energies in an ideal gas follows the exponential law (as a very special case of the chi-squared distribution). If we want to arrive eventually at the Gompertz curve, then we can earn the desired value exponentially by adopting this assumption.

Numerous models have been suggested in the literature that are designed to derive the exponential curve (1) by exploiting exponential assumptions of a 'general nature', such as the Arrhenius law of chemical kinetics, which seems to us to be a reasonable starting point (Strehler 2000, Golubev 2009). However, it has been repeatedly argued (e.g., in Tenenbein and Vanderhoof (1980)) that putting an exponent at the base of the derivation of the Gompertz law is, at least, controversial (Golubev 2009). Starting from the other functional form of the baseline mortality rate (e.g., a constant, as in the reliability theory of aging of Gavrilov and Gavrilova (2001)) should also be justified. As far as we know, the only approach that is free from assumptions of this kind is based on the well-known fact that the Gompertz law is the limiting distribution for the minimum of $n$ i.i.d. random variables when $n \rightarrow \infty$ (Gnedenko 1943; Abernethy 1979, 1998). The application of this result to organisms still needs additional justification, but our personal feeling is that the corresponding limiting procedure most likely lies in the origin of the exponentially increasing force of mortality. Indeed, the organism can, from the point of view of engineering reliability, be considered as a 
series system of a large number $n$ of basic (elementary) components (the basic component can have internal redundancy, including, for example, the possibility of imperfect repair). These components do not necessarily have exponentially distributed lifetimes and can be dependent via mutual resources and the environment. The latter formally violates the independence assumptions of the original limiting theory. However, certain generalizations based on biological and reliability theory concepts may be formulated in the future for the justification of the Gompertz law for $n \rightarrow \infty$.

\section{Assumption 2}

An organism is characterized by its vitality function $V(t), V(0) \equiv V_{0}$, which decreases with age $t$. Yashin et al. (2002), as in the original paper, called this function the maximum capacity of energy supply for an organism at age $t$. It can also obviously be interpreted as the stress resistance of an organism. Death occurs at age $t$, when, for the first time, $Y>V(t)$. We discuss this assumption in conjunction with the last one.

\section{Assumption 3}

The rate $K(t)=K$ is a constant, and the force of mortality is defined as

$$
\mu(t)=K P(Y>V(t))=K e^{-\frac{V(t)}{D}} .
$$

Equation (3) is called a postulate in Strehler and Mildvan (1960). However, it follows from the theory of point processes that (3) is true only when the underlying point process $\left\{T_{i}\right\}$ is the homogeneous Poisson process, and therefore, that the interarrival times of events (stresses) are exponentially distributed. This is a rather stringent condition, which was not pointed out in the original and subsequent papers discussing the SM model. It should also be noted that, while (3) can be generalized to the case of the nonhomogeneous Poisson process with the age-dependent rate $K(t)$, the Poisson property of the underlying process is crucial for the product in the right-hand side of (3), as proved in Finkelstein (2008).

The following observation should be also made: as the force of mortality is a population characteristic, the vitality $V(t)$ should also be understood in this sense. However, it is obviously introduced by Assumption 1 as an individual (stochastic) characteristic. Therefore, we cannot simply substitute it with the expectation, as it is well known that $E\left[e^{-\frac{V(t)}{D}}\right] \neq e^{-\frac{E[V(t)]}{D}}$. 
Thus, while there are a few important deficiencies in the original formulation of the model, it formally leads to properties of mortality rates that are important and justified in practice.

Now we are ready to equate (1) and (3), as our goal is to move from (3) to (1). As in the original paper, we will now show via elementary derivations that $V(t)$ is linearly declining with age. It should be noted that this 'shape' is in line with the current understanding of the decline in the essential biological markers and the corresponding data, at least for the human middle-age span (Golubev 2009). Thus,

$$
\mu(t)=a e^{b t}=K e^{-\frac{V(t)}{D}}
$$

and taking logarithms of both sides $\left(V(0) \equiv V_{0}\right)$ :

$$
V(t)=V_{0}\left(1-\left(b / V_{0}\right) t\right)=V_{0}(1-B t),
$$

where, formally, $B=b / \ln (K / a)=D b / V_{0}$, and this quantity is usually called the individual rate of aging (in contrast with the population rate of aging $b$ ). Substituting (5) into (4):

$$
\mu(t)=a e^{b t}=K e^{-\frac{V_{0}(1-B t)}{D}}=K e^{-\frac{V_{0}}{D}} e^{\frac{V_{0} B t}{D}}
$$

and, thus,

$$
a=K e^{-\frac{V_{0}}{D}} ; \quad b=V_{0} B / D
$$

Comparing two equations for the mortality rate, we see the dependence between $a$ and $b$ (negative correlation): the larger $a$ results in the smaller $b$. From (7), this dependence can be written as

$$
\ln a=\ln K-\frac{1}{B} b,
$$

which is known in the literature as the SM correlation. This correlation has been observed empirically in various human populations. It follows from (8) that 


$$
\ln \mu(t)=\ln a+b t=\ln K+b(t-1 / B)),
$$

meaning that the logarithms of mortality rates for different populations (e.g., with different $a$ ) intersect at one point with coordinates $(\ln K, 1 / B)$. This has been experimentally observed and reported in the literature, although some criticisms and violations of this rule have also been discussed (see, e.g., Yashin et al. (2001), Yashin et al. (2002), and the next section).

At first sight, it seems intriguing that the SM correlation, which is derived using some general, partially unjustified assumptions, complies with the real mortality data. For several decades, the SM correlation was believed to be a kind of universal demographic law valid for both period and cohort mortality data (Yashin et al. 2001). However, it was argued that the SM correlation is partly an artifact of neglecting the constant term in the Gompertz-Makeham model; i.e., of using the pure Gompertz law to treat mortality data derived from populations that actually conform to the GompetzMakeham law (when a constant $c$ is added to the right-hand side of (1)). For modern populations of developed countries, $c$ is usually relatively small and can be neglected, which is not the case for historical populations (e.g., before 1950), for which the original model was claimed. This problem was first recognized and thoroughly discussed by Gavrilov and Gavrilova (1991) (see also Golubev $(2004,2009)$ ). However, we believe further theoretical statistical investigation of this issue is needed.

\section{What 'drives' the SM correlation?}

In this section, we will focus on the changes in $V_{0} / D$, which, according to (7), 'drive' the SM correlation (i.e., the larger $a$ corresponds to the smaller $b$ ). Recall that $V_{0}$ describes the biological 'properties' of organisms (initial resource or resistance), whereas $D$ is the mean demand for energy under stress.

Assume first that $D$ is a constant and $V_{0}$ is a variable for different populations. It follows from equations (5) and (9) that the model predicts the maximum age of $t=1 / B$ for all values of $V_{0}$. Therefore, populations with larger values of resources will obviously have a more rectangularized survival curve than those with smaller values, as the younger ages will benefit more from the increase in $V_{0}$ than the older ages. We can also expect (for developed countries) that $V_{0}$ should increase with chronological time. However, as $V_{0}$ in the latter case can be considered as a genetic parameter, its changes are not likely to be substantial in the course of 50 years or so. 
Let $V_{0}$ be fixed now, whereas $D$ is variable. This assumption can make sense, as, due to the better environment and health care (see later), the amount of energy that is needed for an organism living in a developed country to overcome a stress event decreases with time. It can be seen as a kind of a time-dependent shield-or, alternatively, an as additional supply of energy via medication, etc. - which is obviously improving with time. It should be noted that a similar concept of 'protection' was used by Yashin et al. (2001) (see also Golubev, 2009) in a slightly different context. As in the first case, the decrease in $D$ also leads to the rectangularization of the corresponding survival curves. Finally, when both $V_{0}$ and $D$ are 'improving', $V_{0} / D$ is increasing and the conclusions are the same.

The described patterns were probably the main driving forces for the rectangularization of survival curves for the developed countries in the first half of the $20^{\text {th }}$ century: i.e., the better environment led to reasonable improvements in health care without seriously affecting the oldest old, as more advanced health care was needed to 'save' a noticeable number of very elderly lives (see Section 5). As was discussed above, $K$ and $B$ in the original model are constants. The departure from the rectangularization pattern observed in the developed countries in the second half of the $20^{\text {th }}$ century suggests that this assumption is wrong, and that the corresponding quantities could vary (Yashin et al. 2001). In Section 5, we will consider another model, which is somewhat related to changes in $K$.

\section{The Strehler-Mildvan model and mortality deceleration}

It is well known that the Gompertz law has its limits, and that a more plausible model for the oldest-old mortality would account for the observed mortality deceleration at old ages.

Assume that our population is heterogeneous and that its force of mortality is described by the multiplicative gamma-Gompertz frailty model (Beard 1959; Vaupel et al. 1979). For simplicity of comparison - and, indeed, without loss of generality-let the mean of the frailty parameter equal 1 , which means that the scale parameter of the corresponding gamma distribution is equal to the shape parameter, i.e., $\beta=\alpha$. Then the following form of the population force of mortality can be easily obtained when the baseline force of mortality is given by (1):

$$
\mu(t)=\frac{a e^{b t}}{1+C\left(e^{b t}-1\right)},
$$

where $C=a / b \beta$. 
When $C\left(e^{b t}-1\right)<<1$, which should correspond to human ages less than 100 years, the right-hand side of (10) tends toward the Gompertz model (1). On the other hand, when $t \rightarrow \infty$, as the weakest populations are dying out first, $\mu(t)$ tends to the constant $a / C$, which models the deceleration of mortality at old ages and the corresponding mortality plateau. Analyzing the oldest-old mortality, Gampe (2010) showed that human mortality after the age of 105-110 levels off at the value corresponding to the approximately $50 \%$ annual probability of death. The fixed frailty models have been intensively investigated in the literature (see Finkelstein and Esaulova (2006), Missov and Finkelstein (2011) and Steinsaltz and Wachter (2006) (and references therein) for a general asymptotic theory of mortality deceleration and plateaus). It should be noted that there are other probabilistic models that can produce mortality plateaus-e.g., models based on the first passage times for the Wiener process with drift (Anderson 2000; Li and Anderson 2009) - but that these models are not of the Gompertz type for a finite amount of time, which is a prerequisite for inclusion in our paper. It is also worth mentioning that there are biological explanations for mortality plateaus (Mueller and Rose 1996).

In view of our reasoning in this section, the following question arises: What would happen, if, within the framework of the Srtehler-Mildvan model, instead of using (1), we considered the more realistic relation of (10)? To test this out, let us substitute in equation (4) the Gompertz curve (1) with the 'gamma-Gompertz curve' (10):

$$
\frac{a e^{b t}}{1+C\left(e^{b t}-1\right)}=K e^{-\frac{V(t)}{D}} .
$$

Thus,

$$
V(t)=D \ln \left(\frac{K\left(1+C\left(e^{b t}-1\right)\right)}{a e^{b t}}\right) .
$$

This equation is obviously less appealing than the linear decreasing function (5), but for the age span in which the Gompertz law holds, it reduces to $V(t) \approx V_{0}(1-B t)$. However, for oldest-old mortality,

$$
\lim _{t \rightarrow \infty} V(t) \equiv V_{a}=D(\ln K C / a)>0,
$$

which means that vitality initially decreases linearly in accordance with (5), and then asymptotically approaches (monotonically decreasing) level $V_{a}$. This corresponds to 
the asymptotic mortality plateau, which, as follows from (10), has the value of $a / C$. Therefore, in accordance with (4), the probability of dying at an advanced age under each stress is a time-independent (asymptotically) constant $a / K C$, which also makes sense as the consequence of the corresponding mortality plateau. A similar approach can be found in Arbeev et al. (2005), where the cancer incidence rates were modeled by the modified SM model. The authors used the exponentially decreasing (instead of the linear decreasing of the original SM model) vitality for the homogeneous case, and then incorporated the corresponding baseline incidence rate functions into the gamma-frailty model. The approach of this section is slightly different. Moreover, our main interest is in the shape of the resulting vitality, and, specifically, in its asymptotic plateau.

Obviously, the correlation (8) formally holds for the age span in which the Gompertz law holds, but the end-of life-point $1 / B$ is now out of the picture (indeed, the SM model has been heavily criticized because of this interpretation). Therefore, based on the gamma-Gompertz generalization of the Gompertz model, we have presented a useful modification of the SM model that partially addresses some of the criticisms of the model. It should be noted that Golubev (2009) has developed an interesting generalization of the SM model by partitioning the organisms' resources (vitality) between stress resistance and protection from parametobolic damage.

\section{An alternative approach: Lifesaving $\Rightarrow$ proportional hazards}

As was noted above, the situation changed in the second half of the previous century, as a departure from rectangularization, accompanied by shifts in the corresponding survival functions (due to shifts in mortality rates), were observed (e.g., as in the Bongaarts and Feeney (2002) Gompertz-shift model). This also indicates that the SM correlation is not a universal law, as many authors believed. Yashin et al. (2001) confirmed the validity of these observations, and provided a useful discussion of the possible causes for these changes, focusing mainly on revising the original SM model and generalizing it to the case in which $K$ and (or) $B$ are not constants.

The explanation of the changed patterns of survival is, however, a departure from the original SM model and a consideration of the vitality-independent approach. It is based on the concept of lifesaving: i.e., that the environment not only supplies additional energy under stress, but, due to crucial advances in health care in recent decades, saves lives that previously would have been lost. The stochastic 'lifesaving model' (with a discussion of the necessary assumptions) was developed in Finkelstein (2005, 2008). It should be noted that Vaupel and Yashin (1987) assumed there was a finite number of lifesavings, whereas we were dealing with a random number of these events. 
Consider a lifetime with the (baseline) force of mortality $\mu(t)$. Assume that stress, which occurs in accordance with the corresponding lifetime distribution, is fatal with probability $p(t)$, and 'is cured' with probability $1-p(t)$. Note that the vitality of an organism can be still taken into account if we assume that $1-p(t)$ is a decreasing function of age (an exponentially decreasing pattern could be a good candidate). Thus, in accordance with the lifesaving model (Finkelstein 2005), the initial nonhomogeneous Poisson process of events with rate $\mu(t)$ is terminated (i.e., each event terminates the process with probability $p(t)$ and is 'harmless' with probability $1-p(t))$ to end up with the distribution of time to termination characterized by the mortality rate $p(t) \mu(t)$.

Consider the described general lifesaving model in the demographic context. In accordance with our vitality-free approach, assume now that probability $p(t)$ is not age-dependent; i.e., $p(t) \equiv p$. Obviously, the state of an organism (vitality) can 'affect' this probability, but as in the first approximation, we assume that it does not, as today it is mostly defined by the new 'technical' abilities of treatment, e.g., medical conditions that could not be treated before or the performance of operations that were not previously possible. Note that health conditions leading to death (without lifesaving) obviously occur in accordance with the baseline, increasing with age $\mu(t)$. However, our assumption means that the proportion of conditions that can be now cured does not depend on age, as with the first approximation. Therefore, the resulting force of mortality $p \mu(t)$ follows the proportional hazard $(\mathrm{PH})$ model. Let (1) define the baseline force of mortality for a developed country at, e.g., time $x_{b}=1950$. Then it can be modified for time $x>x_{b}$ to

$$
\mu_{\tau}(t)=p_{\tau} a e^{b t},
$$

where $\tau=x-x_{b}$ and $p_{\tau}$ is constant in age for the fixed $\tau$. Thus, the environment, due to lifesaving, 'decreases only parameter $a$ ', without affecting the slope of the logarithmic mortality rate $b$. This perfectly complies with the Gompertz shift model of Bongaarts and Feeney (2002) and with other experimental studies. It can also explain the change in the rectangularization pattern to shifts in the corresponding survival curves for developed countries in recent decades. Note that the assumption of the underlying Gompertz law is essential for the described change in the pattern, which is easily seen from $(11)$, as $p_{\tau}=e^{\ln p_{\tau}}\left(\ln p_{\tau}<0\right)$ creates shifts in age for the baseline mortality rate. It is also worth mentioning that, although the method of 'constructing' 
the resulting mortality rate in the SM model, which is captured by equation (3), formally resembles our lifesaving approach, the difference lies in the fact that the corresponding probabilities are 'applied' to each stress event in the former case, and to events occurring in accordance with the non-homogeneous Poisson process with rate $\mu(t)$ in the latter.

As we mentioned above, in reality $p(t)$ is likely to be a (slowly) increasing function. The larger values of $p(t)$ (for all values of $t$ ) obviously correspond to the more rectangularized survival function. With health care advances, this effect is likely to decrease in chronological time, which can be considered as another possible explanation for the departures from the rectangularization pattern.

\section{Discussion}

Every model is useful to the extent that it explains real phenomena. For several decades, the SM correlation was believed to be a kind of a universal demographic law valid for both period and cohort mortality data. However, the mortality data of recent decades exhibit departures from this pattern, which means that the law either needs modifications, or that it should no longer be considered a useful tool for analyzing contemporary mortality data.

We started our discussion by revisiting the foundations of the SM model, and stating that the original Assumption 3 holds only for the Poisson process of stresses. It follows from the theory of stochastic point processes that the rate $K$ alone does not completely define the point process (this is true only for the Poisson process). The fact that the SM correlation was consistent with the mortality data for several decades implicitly shows that the process of shocks (demands) can be well-approximated by the corresponding Poisson process. We also acknowledge that the unjustified original Assumption 1 actually implants the desired exponentiality into the model. However, in spite of all of these stated deficiencies, the model was effective for a number of decades.

In addition to discussing the assumptions and limitations of the original SM model, we have suggested some modifications and new approaches that can account for the changing pattern of mortality data, and, specifically, for oldest-old mortality. In reality, we are performing a very simple operation: instead of equating the Gompertz mortality rate $a e^{b t}$ to $K e^{-\frac{V(t)}{D}}$, and arriving at the linear $V(t)$, as in the original SM model; we are equating to $K e^{-\frac{V(t)}{D}}$, the mortality rate (logistic) that results from the gamma- 
Gompertz frailty model. In this way, we gain some useful properties (e.g., the shape of $V(t)$, which is of the main interest here), and obviously lose the simplicity of the original model. It should be noted that we do not assume from the beginning that $V(t)=V_{0}(1-B t)$ and then obtain $a$ and $b$ for the resulting $a e^{b t}$ as functions of parameters involved, which is usually done for the sake of statistical inference. Therefore, we suggest a possible approach to understanding the shape of the resulting mortality plateau that also eliminates the 'end-of-life point'. This definitely merits further study and statistical analysis. Thus, at this stage, our approach remains rather formal, and is limited to pointing out one of the possible ways of dealing with the shortcomings of the SM model.

Another possible approach for dealing with heterogeneity in the context of mortality modeling can be found in Anderson et al. (2008), in which the authors presented an analytical model for the average vitality trajectory that begins by linearly decreasing and asymptotically approaching a zero-vitality boundary. Although the corresponding equation for the average $V(t)$ is complex, it can, in principle, be used in a manner similar to our model in Section 5, but by assuming this shape from the beginning. As $V(t)$ initially decreases linearly, the Gompertz mortality curve shape also holds initially, but then, as $V(t)$ vanishes at the infinity, we do not arrive at the plateau. Moreover, this is an example of an approach that considers the changing (or evolving) heterogeneity ( $\mathrm{Li}$ and Anderson 2009), whereas our approach deals with the fixed frailty. Heterogeneity in the latter case is modeled by the multiplicative (proportional hazards) model, where the fixed frailty parameter 'acts directly on the mortality rate'. It should be noted that mortality plateaus can also result in the vitalitybased models with evolving heterogeneity. However, this happens when individual aging (i.e., the vitality trajectories) is modeled by the corresponding Wiener process with positive drift, and death occurs when the given vitality boundary is reached for the first time.

A recent paper by Zheng, Yang, and Land (2011) also addressed heterogeneity in the context of the SM model. It contains impressive statistical information on mortality rates for different countries and periods of time. The heterogeneity in lifetimes considered in that empirical study was due to the randomness in the SM parameters, whereas we analyzed the shape of the mortality rate from the modeling point of view.

Most probably, a better fit to mortality data could be obtained by using the combination of the SM model with the vitality-independent approach suggested in the current paper. This 'mixture' may constitute a topic for future research. 


\section{Acknowledgements}

The author would like to thank three anonymous reviewers and the Associate Editor for their extremely helpful and insightful comments. 


\section{References}

Abernethy, J.D. (1979). The exponential decrease in mortality rate with age attributed to wearing-out of biological components. Journal of Theoretical Biology 80(3): 333-354. doi:10.1016/0022-5193(79)90098-5.

Abernethy, J.D. (1998). Gompertzian mortality originates in the winding-down the mitotic clock. Journal of Theoretical Biology 192(4): 419-435. doi:10.1006/ jtbi.1998.0657.

Anderson, J.J. (2000). A vitality-based model relating stressors and environmental properties to organism survival. Ecological Monographs 70(3): 445-470. doi:10.1890/0012-9615(2000)070[0445:AVBMRS]2.0.CO;2.

Anderson, J.J., Gildea, M.C., Williams, D.W., and Li, T. (2008). Linking growth, survival, and heterogeneity through vitality. The American Naturalist 171(1): E20-E43. doi:10.1086/524199.

Arbeev, K.G., Ukraintseva, S.V., Arbeeva, L.S., and Yashin, A.I. (2005). Mathematical models for human cancer incidence rates. Demographic Research 12(10): $237-$ 272. doi:10.4054/DemRes.2005.12.10.

Beard, R.E. (1959). Note on some mathematical mortality models. In: Woolstenholme, G. and O'Connor, M. (eds.). The Lifespan of Animals. Boston: Little, Brown and Company: 302-311.

Bongaarts, J. and Feeney, G. (2002). How long do we live? Population and Development Review 28(1): 13-29. doi:10.1111/j.1728-4457.2002.00013.x.

Finkelstein, M. (2005). Lifesaving explains mortality decline with time. Mathematical Biosciences 196(2): 187-197. doi:10.1016/j.mbs.2005.04.004.

Finkelstein, M. (2008). Failure Rate Modeling for Reliability and Risk. London: Springer.

Finkelstein, M. and Esaulova, V. (2006). Asymptotic behaviour of a general class of mixture failure rates. Advances in Applied Probability 38(1): 244-262. doi:10.1239/aap/1143936149.

Gampe, J. (2010). Human mortality beyond age 110. In: Maier,H., Gampe, J., Jeune, B., Robine, J.-M., and Vaupel, J.W. (eds.). Supercentenarians. Demographic Research Monographs. Berlin: Springer: 219-230.

Gavrilov, L.A. and Gavrilova, N.S. (1991). The Biology of Life Span: A Quantitative Approach. New York: Harwood Academic Publisher. 
Gavrilov, L.A. and Gavrilova, N.S. (2001). The reliability theory of ageing and longevity. Journal of Theoretical Biology 213(4): 527-545. doi:10.1006/ jtbi.2001.2430.

Gnedenko, B.V. (1943). Sur la distribution limite du terme maximum d'une serie aleatore. The Annals Of Mathematics 44(3): 423-453. doi:10.2307/1968974.

Golubev, A. (2004). Does Makeham make sense? Biogerontology 5(3): 159-167. doi:10.1023/B:BGEN.0000031153.63563.58.

Golubev, A. (2009). How could the Gompertz-Makeham law evolve. Journal of Theoretical Biology 238(1): 1-17. doi:10.1016/j.jtbi.2009.01.009.

Gompertz, B. (1825). On the nature of the function expressive of the law of human mortality and on a new mode of determining the value of life contingencies. Philosophical Transactions of the Royal Society 115: 513-585. doi:10.1098/rstl.1825.0026.

Li, T. and Anderson, J.J. (2009). The vitality model: A way to understand population survival and demographic heterogeneity. Theoretical Population Biology 76(2): 118-131. doi:10.1016/j.tpb.2009.05.004.

Makeham, W.M. (1860). On the law of mortality and the construction of annuity tables. The Assurance Magazine and Journal of the Institute of Actuaries 8(6): 301-310.

Missov, T.I. and Finkelstein, M. (2011). Admissible frailty distributions for a general class of mixture mortality models with known asymptotics. Theoretical Population Biology 80(1): 64-70. doi:10.1016/j.tpb.2011.05.001.

Mueller, L.D. and Rose, M.R. (1996). Evolutionary theory predicts late-life mortality plateaus. PNAS, 93(26): 15249-15253. doi:10.1073/pnas.93.26.15249.

Steinsaltz, D.R., and Wachter, K.W. (2006). Understanding mortality rate deceleration and heterogeneity. Mathematical Population Studies 13(1): 19-37. doi:10.1080/ 08898480500452117.

Strehler, B.L. (2000). Understanding aging. Methods in Molecular Medicine 38(1): 1-19. doi:10.1385/1-59259-070-5:1.

Strehler, B.L., and Mildvan, A.S. (1960). General theory of mortality and aging. Science 132(3428): 14-21. doi:10.1126/science.132.3418.14.

Tenenbein, A. and Vanderhoof, I.T. (1980). New mathematical laws of select and ultimate mortality. Trans. Soc. Actuaries 32: 119-170. 
Vaupel, J.W. and Yashin, A.I. (1987). Repeated resuscitation: How life saving alters life tables. Demography 24(1): 123-135. doi:10.2307/2061512.

Vaupel, J.W., Manton, K.G., and Stallard, E. (1979). The impact of heterogeneity in individual frailty on the dynamics of mortality. Demography 16(3): 439-454. doi:10.2307/2061224.

Yashin, A.I., Begun, A.S., Boiko, S.I., Ukraintseva, S.V., and Oeppen, J. (2001) The new trends in survival improvement require a revision of traditional gerontological concepts. Experimental Gerontology 37(1): 157-167. doi:10.1016/S0531-5565(01)00154-1.

Yashin, A.I., Ukraintseva' S.V., Boiko, S.I., and Arbeev, K.G. (2002). Individual aging and mortality rate: How are they related. Social Biology 49(3-4): 206-217. doi:10.1080/19485565.2002.9989059.

Zheng, H., Yang, Y., and Land, K.C. (2011). Heterogeneity in the Strehler-Mildvan General Theory of mortality and aging. Demography 48(1): 267-290. doi:10. 1007/s13524-011-0013-8. 